\title{
Apoptotic induction of lung adenocarcinoma A549 cells infected by recombinant RVG Newcastle disease virus (rL-RVG) in vitro
}

\author{
YULAN YAN ${ }^{1}$, BING LIANG $^{2}$, JIN ZHANG $^{1}$, YANG LIU $^{1}$ and XUEFENG BU ${ }^{3}$ \\ ${ }^{1}$ Department of Respiratory Medicine, Affiliated People's Hospital of Jiangsu University, Zhenjiang, Jiangsu 212002, \\ ${ }^{2}$ Department of Internal Medicine, Clinical Medicine College of Jiangsu University, Zhenjiang, Jiangsu 212013; \\ ${ }^{3}$ Department of General Surgery, Affiliated People's Hospital of Jiangsu University, Zhenjiang, Jiangsu 212002, P.R. China
}

Received November 29, 2013; Accepted August 22, 2014

DOI: $10.3892 / \mathrm{mmr} .2014 .2657$

\begin{abstract}
Newcastle disease virus (NDV) is a member of the genus Avulavirus in the Paramyxoviridae family and its antitumor properties depend on its ability to kill malignant cells while not affecting normal cells. The present study investigated a recombinant avirulent NDV LaSota strain (wild-type NDV strain) expressing the rabies virus glycoprotein (rL-RVG), examined its oncolytic effect on the lung adenocarcinoma A549 cell line and evaluated its potential to serve as a vaccine against lung cancer. A549 cells were infected with the rL-RVG virus and analyzed by MTT, western blot, polymerase chain reaction (PCR), immunofluorescence, terminal deoxynucleotidyl transferase dUTP nick end labeling and flow-cytometric analyses. PCR, western blot and immunofluorescence showed that the RVG gene and protein were stably expressed in A549 cells following infection with rL-RVG. The growth of A549 cells in the rL-RVG group was inhibited more effectively compared to those infected with the wild-type NDV strain. MTT results showed that cell growth inhibition rates in the rL-RVG group were significantly higher than those in the NDV group $(\mathrm{P}<0.05)$. Early apoptosis in the rL-RVG group was also more evident, with the apoptotic index being increased in rL-RVG group. The expression of the pro-apoptotic proteins caspase-3, -8 and -9 increased. The expression of caspase-3 decreased following application of the broad-specificity caspase inhibitor Z-VAD-FMK. However, the expression of the inhibitory apoptosis protein B-cell lymphoma 2 (bcl-2) did not change, but bcl-2-associated $\mathrm{X} / \mathrm{bcl}-2$ ratio was higher in the rL-RVG group than that in the NDV group. The rL-RVG strain was able to suppress lung cancer cell growth and promote lung cancer cell apoptosis to a greater extent than
\end{abstract}

Correspondence to: $\mathrm{Mr}$ Xuefeng $\mathrm{Bu}$, Department of General Surgery, Affiliated People's Hospital of Jiangsu University, 8 Dianli Road, Zhenjiang, Jiangsu 212002, P.R. China

E-mail: xuefengbu05@163.com

Key words: apoptosis, lung adenocarcinoma, oncolytic, recombinant Newcastle disease virus, rL-rabies virus glycoprotein the wild-type NDV strain. Therefore, the rL-RVG strain is a potent antitumor agent.

\section{Introduction}

Lung cancer is the leading cause of mortality worldwide, being the cause of $>1,000,000$ mortalities yearly (1). In 2013, an estimated 82 males and 38 females per 100,000 will die from lung cancer (2). Currently, surgery, radiotherapy and chemotherapy serve as the most common treatments in cancer therapy. In a large number of cases, patients get diagnosed with cancer past the time-point of surgical treatment being the most promising treatment method. Moreover, conventional therapies, including radiotherapy and chemotherapy, frequently have severe side effects as they do not selectively kill cancer cells but also normal cells (3). Therefore, it is important to discover novel treatments for lung cancer.

Oncolytic therapy is a novel biological treatment integrating gene and immune therapy, which is widely studied all over the world. Oncolytic virotherapy is a promising form of gene therapy for cancer, employing nature's own agents to identify and destroy malignant cells (4). Newcastle disease virus (NDV) is one of these oncolytic viruses. The first study on NDV being applied to treat uterine carcinoma dates back to the early 1950s (5). Since then NDV has been studied as an ideal candidate for cancer therapy. NDV has numerous advantages making it an attractive viral vector to cure cancer. For example, NDV has the ability to selectively kill malignant cells, while not targeting normal cells. It also has the ability to replicate in cancer cells, with a 10,000 fold higher efficiency opposed to that in normal cells (5).

NDV is a non-segmented negative-strand RNA virus, which is part of the genus Avulavirus from the Paramyxoviridae family. According to their pathogenic properties, NDVs can be divided into three major pathotypes: Lentogenic strains, mesogenic strains and velogenic strains $(6,7)$, while the LaSota NDV strain (or wild-type NDV strain) is a lentogenic strain, which causes clinically insignificant or inapparent respiratory disease. The RNA of the NDV genome encodes six major structural proteins: Nucleoprotein (NP), phosphoprotein (P), matrix protein $(\mathrm{M})$, fusion protein $(\mathrm{F})$, haemagglutinin-neuraminidase (HN) and large (L) RNA-dependent RNA polymerase, with the six genes being in the order 3'-NP-P-M-F-HN-L-5' (8). 
The infectious ability of NDV depends on hemagglutinin-neuraminidase $(\mathrm{HN})$ and fusion $(\mathrm{F})$ glycoproteins. The two glycoproteins are embedded in the viral lipid membrane. The F glycoprotein precursor (F0) is proteolytically cleaved to F1 and F2 (4). Finally, the penetration of NDV to target cells is completed by the endocytosis process (9).

The rabies virus (RV) is an enveloped, bullet-shaped virus, which belongs to the genus Lyssavirus of the family Rhabdoviridae. The RV genome is composed of 3'-N-P-M-G-L-5', encoding nucleoprotein (N), phosphoprotein $(\mathrm{P})$, matrix protein $(\mathrm{M})$, glycoprotein $(\mathrm{G})$ and RNA polymerase (L) (10). The rabies virus glycoprotein (RVG) has been associated with apoptosis of host cells. Préhaud et al (11) showed that the Evelyn Rokitnicki Abelseth (ERA) strain of $\mathrm{RV}$ triggers the caspase-dependent apoptosis of human cells. It is the induction of $\mathrm{G}$ protein expression instead of $\mathrm{N}$ protein expression that resulted in apoptosis. RV was used as the first virus to be assessed for the treatment of 30 patients with melanomatosis, eight of whom showed tumor regression in experimental settings, and in further clinical trials during the 1950s and 1960s (4).

The rL-RVG used in the present study was provided by the Harbin Veterinary Research Institute (13). The RVG was inserted at the position between the $\mathrm{P}$ and $\mathrm{M}$ genes. rL-RVG retained its titers of up to $10^{9.8} 50 \%$ egg infective doses $\left(\operatorname{EID}_{50}\right) / \mathrm{ml}$ of allantoic fluid. rL-RVG has the ability to spread from cell to cell and to alter its self-replication in cell culture (12). Therefore, it was hypothesized that the rL-RVG had the ability to self-enhance its effect on cancer cells. Since the mechanisms of rL-RVG to kill cancer cells are not well understood, the objective of the present study was to explore the oncolytic effect of rL-RVG on A549 cells in vitro. The findings indicated that rL-RVG may be used as a novel biotherapeutic drug for lung cancer treatment.

\section{Materials and methods}

The recombinant NDV strain, the wild-type NDV LaSota strain and the anti-NDV antibody were kindly provided by the Harbin Veterinary Research Institute (Harbin, China), which were stored at $-70^{\circ} \mathrm{C}$. All viruses were grown and titrated in A549 cells in 96-well plates. Infection of viruses in A549 cells was confirmed by using an indirect immunofluorescence assay: NDV infection of A549 cells were grown in 96-well plates, cells were fixed in ice-cold $4 \%$ paraformaldehyde in phosphatebuffered saline (PBS) for 15 min at room temperature and subsequently washed with PBS, three times. Cells were blocked in PBS containing $1 \%(\mathrm{wt} / \mathrm{vol})$ bovine serumalbumin (BSA) at $4^{\circ} \mathrm{C}$ for $1 \mathrm{~h}$. Cells were then incubated with chicken antibody against NDV for $30 \mathrm{~min}$ at room temperature, washed three times with PBS containing $0.05 \%$ Tween 20 and stained with a Cy3-conjugated goat anti-chicken antibody for $30 \mathrm{~min}$. Following this, the cells were washed three times with PBS, and their nuclei stained with Hoechst 33342. Cells were subsequently analyzed under an immunofluorescence microscope (Axio Observer A1; Carl Zeiss, Oberkochen, Germany). Viral titration results were calculated by using the method of Reed and Muench (12). The lung adenocarcinoma cell line A549 was purchased from the Cell Culture Center of the Basic Institute of Medical Sciences, Peking Union Medical College
(Peking, China). A549 cells were cultured in 24 well-plates with Dulbecco's modified Eagle' medium (DMEM; Gibco Life Technologies. Carlsbad, CA, USA)) containing 10\% fetal bovine serum culture medium, at $37^{\circ} \mathrm{C}$ and $5 \% \mathrm{CO}_{2}$ incubator for cultivation. The cell culture reagents were obtained from Gibco-BRL (Invitrogen Life Technologies, Carlsbad, CA, USA). The 96-well plates, 24-well plates and six-well plates were purchased from Costar Corning (Corning, NY, USA). All polymerase chain reaction (PCR) primers were synthesized by the Shanghai Sangon Biological Engineering Technology \& Services Co. Ltd (Shanghai, China). The TRIzol reagent was purchased from Invitrogen Life Technologies. The PCR master mix was obtained from Toyobo (Osaka, Japan). The terminal deoxynucleotidyl transferase-mediated dUTP-biotin nick end-labeling (TUNEL) assay kit was obtained from Nanjing Kaiji Biotechnology Development Co. Ltd., (Nanjing, China). The antibody to chicken was purchased from MedImmune, LLC (Gaithersburg, MD, USA). The mouse anti-RV monoclonal antibody and caspase inhibitor Z-Val-Ala-Asp-fluoromethylketone (Z-VAD-FMK) were purchased from Santa Cruz Biotechnology, Inc. (Santa Cruz, CA, USA). The rabbit anti-Caspase-3, 8, 9 monoclonal antibodies, rabbit B-cell lymphoma-2 (bcl-2)-associated X (bax) and mouse anti-bcl-2 monoclonal antibodies were purchased from Boster (Wuhan, China). The horseradish peroxidase (HRP)-conjugated goat anti-rabbit, HRP-conjugated goat anti-mouse antibody, Cy3-conjugated goat anti-rabbit and the fluorescein isothiocyanate (FITC)-conjugated goat anti-mouse antibody were purchased from CoWin Biotech, Co., Ltd. (Beijing, China). The HRP-AffiniPure rabbit anti-chicken was purchased from EarthOx Life Science (Millbrae, CA, USA).

Reverse transcription PCR (RT-PCR) analysis. Total RNA was extracted from lung adenocarcinoma A549 cells by using TRIzol. The cDNA was synthesized with Oligo (dT) primers (Takara Bio, Inc., Otsu, Japan). and Moloney murine leukemia virus reverse transcriptase (Takara Bio, Inc.). The 1/10 cDNA was used for PCR amplification. The PCR protocol was as follows: Initial denaturation at $94^{\circ} \mathrm{C}$ for $5 \mathrm{~min}$, followed by 30 cycles at $94^{\circ} \mathrm{C}$ for $30 \mathrm{sec}$, annealing at $55^{\circ} \mathrm{C}(\mathrm{NDV})$ or $58^{\circ} \mathrm{C}$ for $30 \mathrm{sec}$, and extension at $72^{\circ} \mathrm{C}$ for $30 \mathrm{sec}$ (RVG). The final extension was performed by an incubation step at $72^{\circ} \mathrm{C}$ for $10 \mathrm{~min}$. The PCR products were subjected to electrophoresis in agarose gel (Sigma-Aldrich, St. Louis, MO USA) and visualized with ethidium bromide (Sigma-Aldrich). The bands were analyzed with Quantity One software, version 4.62 (Bio-Rad, Hercules, CA, USA). All primers used are listed in Table I.

Western blot analysis. Western blotting was performed to determine the protein of NDV, RVG, bax, bcl-2 as well as caspase-3, -8 and -9 expression using the antibodies described above antibodies at a dilution of 1:200. A549 cells were infected with rL-RVG and NDV at a multiplicity of infection (MOI) of 10 (11). Whole cell lysates were prepared with radioimmunoprecipitation assay buffer (KangChen Biotech Inc., Shanghai, China) containing protease inhibitor (phenylmethanesulfonyl fluoride) and sodium orthovanadate, and $10 \mu \mathrm{l}$ of each was loaded on a gel and the blot was run under reducing conditions. The membrane 
Table I. Primers for polymerase chain reaction amplification.

\begin{tabular}{ll}
\hline Primer & \multicolumn{1}{c}{ Sequence } \\
\hline rL-RVG & \\
Upstream & Product size (bp) \\
Downstream & 5' AGCCGATGCTCACTACAAG 3' \\
NDV & \\
Upstream & 5' CTGGACGGTTTGGTGGGAA 3' \\
Downstream & 5' TAATGCGACTGCGGGATGTG 3' \\
GAPDH & \\
Upstream & 5' CAAGGTCATCCATGACAACTTTG 3' \\
Downstream & 5' GTCCACCACCCTGTTGCTGTAG 3' \\
\hline
\end{tabular}

rL-RVG, LaSota NDV strain expressing rabies virus glycoprotein; NDV, Newcastle disease virus.

was blocked for 1 h using $5 \%$ bovine serum albumin (BSA) prior to incubation with chicken antibody against NDV (1:300) and mouse antibody against RVG (1:200) overnight at $4^{\circ} \mathrm{C}$. Antibody binding was quantified using an anti-chicken immunoglobulin (Ig)Y antibody or a goat anti-mouse IgG conjugated to HRP at 1/10,000 dilution and visualized using an enhanced chemiluminescence development solution, the Luminata TM Crescendo Western HRP Substrate (Millipore, Middlesex, MA, USA).

Immunofluorescence analysis. Immunofluorescence analysis aimed to detect NDV and RVG protein expression in infected A549 cells. A549 cells were seeded in 24-well plates with $5 \times 10^{4}$ per well, and the next day they were infected with NDV or rL-RVG at an MOI of 10 (11), while the negative group was treated with phosphate-buffered saline (PBS). At $24 \mathrm{~h}$ post-infection, cells were fixed in ice-cold $4 \%$ paraformaldehyde (Solar Biotech Inc., Beijing, China) overnight at $4^{\circ} \mathrm{C}$, then cells were washed three times for $15 \mathrm{~min}$ each with ice-cold PBS. Cells were blocked in $3 \% \mathrm{BSA}$ at $4^{\circ} \mathrm{C}$ for $1 \mathrm{~h}$. Following incubation with chicken antibody against NDV (1:300) and mouse antibody against RVG (1:200) for $1.5 \mathrm{~h}$ at room temperature, cells were then washed three times with PBS. Next, cells were stained with Cy3-conjugated goat anti-chicken (1:300) or FITC-conjugated goat anti-mouse secondary antibody $(1: 100)$ for $30 \mathrm{~min}$ at room temperature, and were washed three times with PBS. Finally, nuclei were stained with Hoechst 33342 (10 $\mu \mathrm{g} / \mathrm{ml}$; Sigma-Aldrich) for $10 \mathrm{~min}$. Cells were analyzed with a confocal laser microscope (Axio Observer A1; Zeiss, Germany).

MTT assays. rL-RVG (109.8EID50/ml) and wild-type NDV strain (109.8EID50 /ml ) were diluted 103, 104, 105 and 106 times by serum-free DMEM. A549 in the logarithmic growth phase were plated at 1x104 cells/well in 96-well plates, incubated overnight, infected by the diluted rL-RVG or NDV. DMEM containing 2\% fetal bovine serum (FBS) was added to the cells of the NDV and rL-RVG groups on the following day. The PBS group was used as a negative control. Then, $20 \mu \mathrm{l}$ MTT $(5 \mathrm{mg} / \mathrm{ml})$ was added into each well. After $4 \mathrm{~h}$ of incubation, $150 \mu 1 \mathrm{DMSO}$ solution was added to each well and plates were agitated for $10 \mathrm{~min}$. Finally, the absorbance was read at $490 \mathrm{~nm}$ wavelength using standard spectrophotometer (FLX800; Bio-tek instruments Inc., Shelburne, VT, USA). The above MTT experiment was repeated three times and the tumor cell growth inhibition rate was calculated. Simultaneously, an inverted phase contrast microscope (ECLIPSE TS100; Nikon, Japan) was used to observe the morphological changes of cells following infection with rL-RVG and wild-type NDV following 24, 48 and $72 \mathrm{~h}$.

Flow cytometric analysis. Flow cytometry was applied to examine early apoptotic cells. A549 cells were infected with wild-type NDV and rL-RVG in six-well plates containing $1 \times 10^{5}$ cells/well. Infected cells were harvested $24,48,72 \mathrm{~h}$ later and washed twice with ice-cold PBS. Cells were then resuspended in $1 \mathrm{X}$ binding buffer from the BD Pharmingen FITC Annexin V Apoptosis Detection Kit I (Becton Dickinson, Franklin Lakes, NJ, USA) at a concentration of $1 \times 10^{5}$ cells/ $\mathrm{ml}$. An aliquot of $100 \mu \mathrm{l}$ of the solution was transferred to a 5-ml culture tube. According to the manufacturer's instructions, $5 \mu 1$ Annexin V-FITC and $5 \mu 1$ propidium iodide (PI) solution were added. Cells were then incubated for $20 \mathrm{~min}$ at room temperature in the dark, followed by addition of $400 \mu \mathrm{l}$ $1 \mathrm{X}$ binding buffer to each tube. Infected cells were analyzed by flow cytometry (Facscalibur, Becton Dickenson). All results were repeated three times.

TUNEL assay. Cells in the logarithmic growth phase were infected with the RVG and NDV and following $48 \mathrm{~h}$ cells were fixed. Initially, the cover slips were in 24 -well plates, and subsequently, cells were grown on cover slips by micropipetter, add rL-RVG and NDV, at $48 \mathrm{~h}$ postinfection, cells were fixed in $4 \%$ formaldehyde for $20 \mathrm{~min}$ at room temperature $\left(15-25^{\circ} \mathrm{C}\right)$. This was followed by rinsing with PBS for $30 \mathrm{~min}$ and incubation with $3 \%$ hydrogen peroxide in methanol for $10 \mathrm{~min}$ at room temperature. After 25-min washes with PBS, the samples were incubated with $0.1 \%$ Triton $\mathrm{X}-100$ and $0.1 \%$ sodium citrate in water for $30 \mathrm{~min}$ at room temperature. For negative control, TdT was not added to the sample, and for positive control, cells were treated by DNase I. After two hours of washing with PBS, pretreated specimens were incubated with $501 \mathrm{~L}$ TdT labeling reaction buffer at $4^{\circ} \mathrm{C}$ overnight in dark and 
A

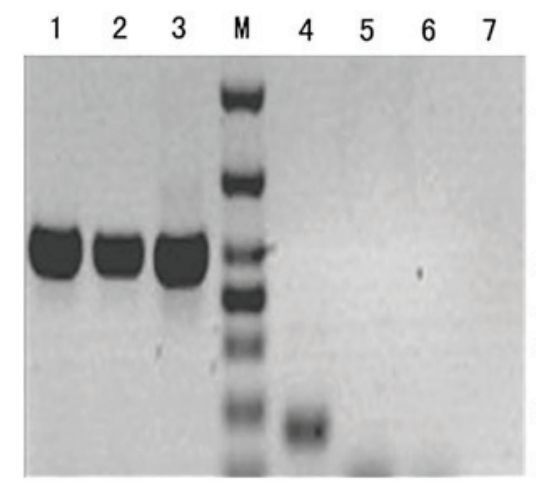

a

B

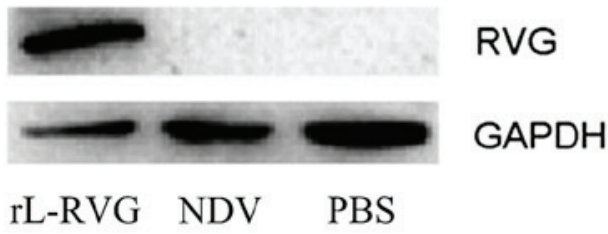

a

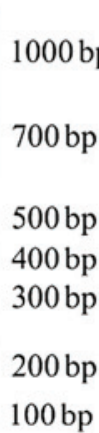

$100 \mathrm{bp}$
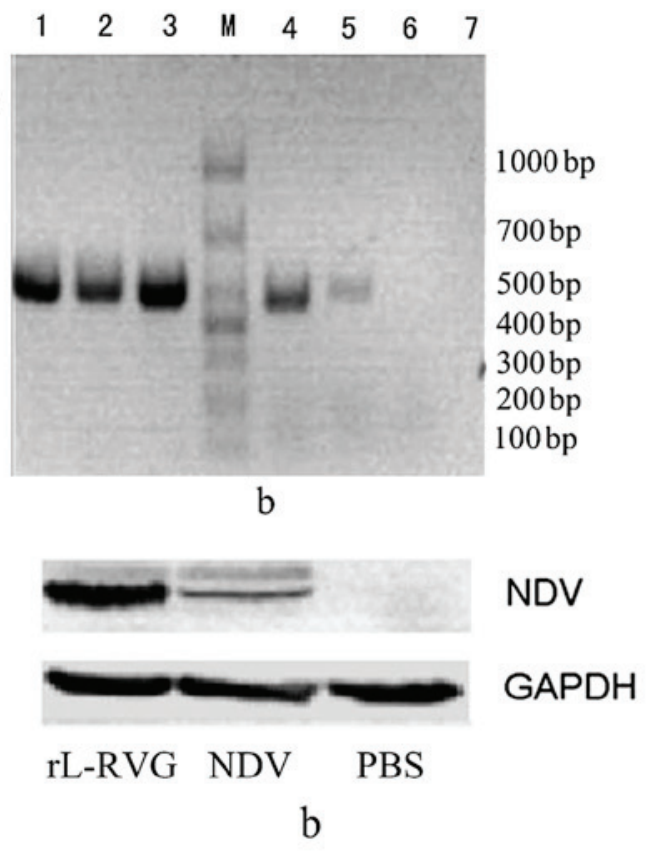

C

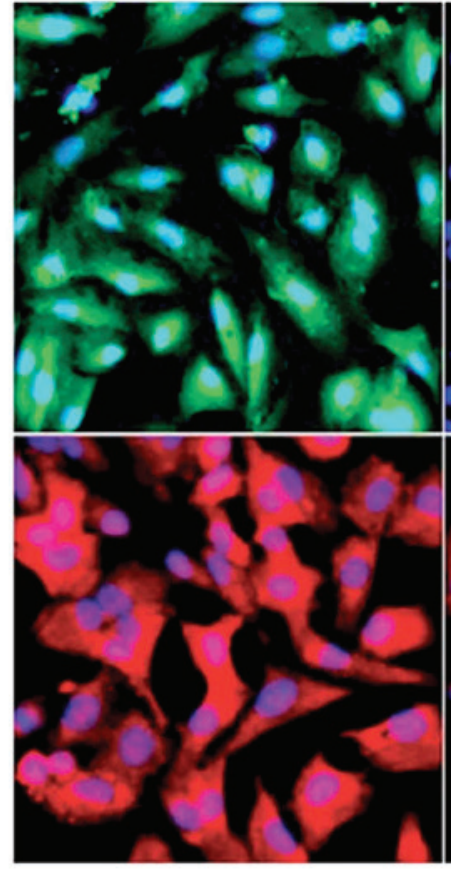

rL-RVG
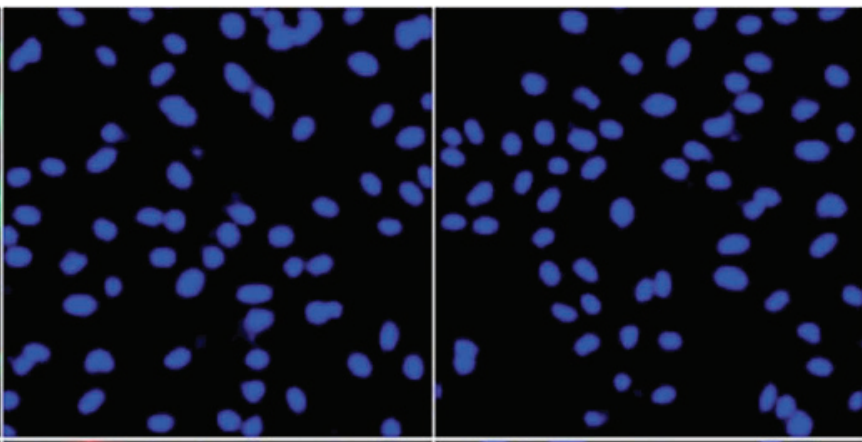

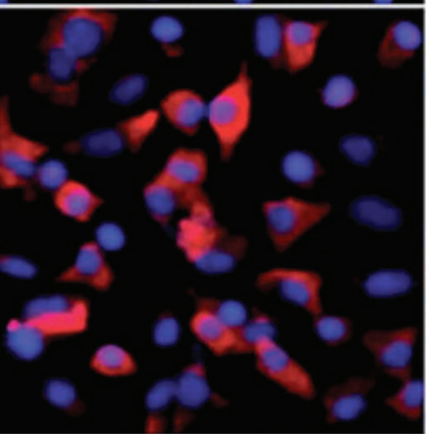

NDV

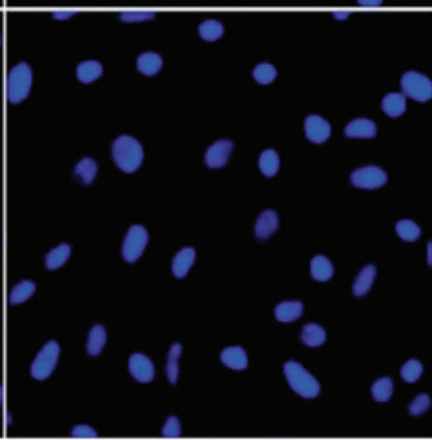

PBS

Figure 1. Expression of the virus in A549 cells. (A) a, Expression of RVG-mRNA; b, expression of HN-mRNA. Lanes: 1, GAPDH in PBS group; 2, GAPDH in NDV group; 3, GAPDH in rL-RVG group; M, maker; 4, rL-RVG group; 5, NDV group; 6, PBS group; 7, blank. (B) Expression of RVG and NDV protein in A549 cells by western blot analysis. Expression of RVG protein was present in the rL-RVG group, and NDV protein was expressed in the rL-RVG and NDV groups. The proteins were not expressed in the PBS group. (C) Immunofluorescence analysis of RVG and NDV protein expression (magnification, $\mathrm{x} 200$ ). The RVG protein (green, top row) was only expressed in the rL-RVG group, while the NDV protein (red, bottom row) was expressed in the rL-RVG and NDV groups. Magnification, x200. rL-RVG, LaSota NDV strain expressing rabies virus glycoprotein; NDV, Newcastle disease virus; PBS, phosphate-buffered saline.

then in a humidified at mosphere at $37^{\circ} \mathrm{C}$ for a further $2-3$ hours. Subsequently, the slides were incubated with 501L streptavidin-HRP for $60 \mathrm{~min}$, followed by detection with $501 \mathrm{~L}$ diaminobenzidine reagent for $10 \mathrm{~min}$. Apoptosis was analyzed using the TUNEL assay kit according to the manufacturer's instructions. Cells were observed and photographed under an optical microscope (ECLIPSE TS100; Nikon, Japan). Areas were selected where the apoptotic cells were distributed evenly, and the number of positive, cells out of 100 cells was counted. This was repeated three times The apoptotic index (AI) was calculated by the number of apoptotic cells/(the number of apoptotic cells + the number of non-apoptotic cells) x100\%.

Statistical analysis. Data were analyzed by SPSS 16.0 software (International Business Machines, Armonk, NY, USA) 
Table II. Viral infection of A549 cells 48 h MTT results of cell growth inhibition rate.

\begin{tabular}{lrrr}
\hline Dilution & NDV, $\%$ & rL-RVG, $\%^{\mathrm{a}}$ & t-value \\
\hline $10^{-3}$ & $47.70 \pm 0.27$ & $67.87 \pm 0.25$ & 16.053 \\
$10^{-4}$ & $39.80 \pm 0.31$ & $43.03 \pm 0.29$ & 14.422 \\
$10^{-5}$ & $17.83 \pm 0.41$ & $32.13 \pm 0.72$ & 5.339 \\
$10^{-6}$ & $3.60 \pm 1.41$ & $18.20 \pm 0.27$ & 15.522 \\
\hline
\end{tabular}

Cell growth inhibition rates were significantly higher in the rL-RVG group of than those in the NDV group (P<0.05). rL-RVG, LaSota NDV strain expressing rabies virus glycoprotein; NDV, Newcastle disease virus.

\section{A}
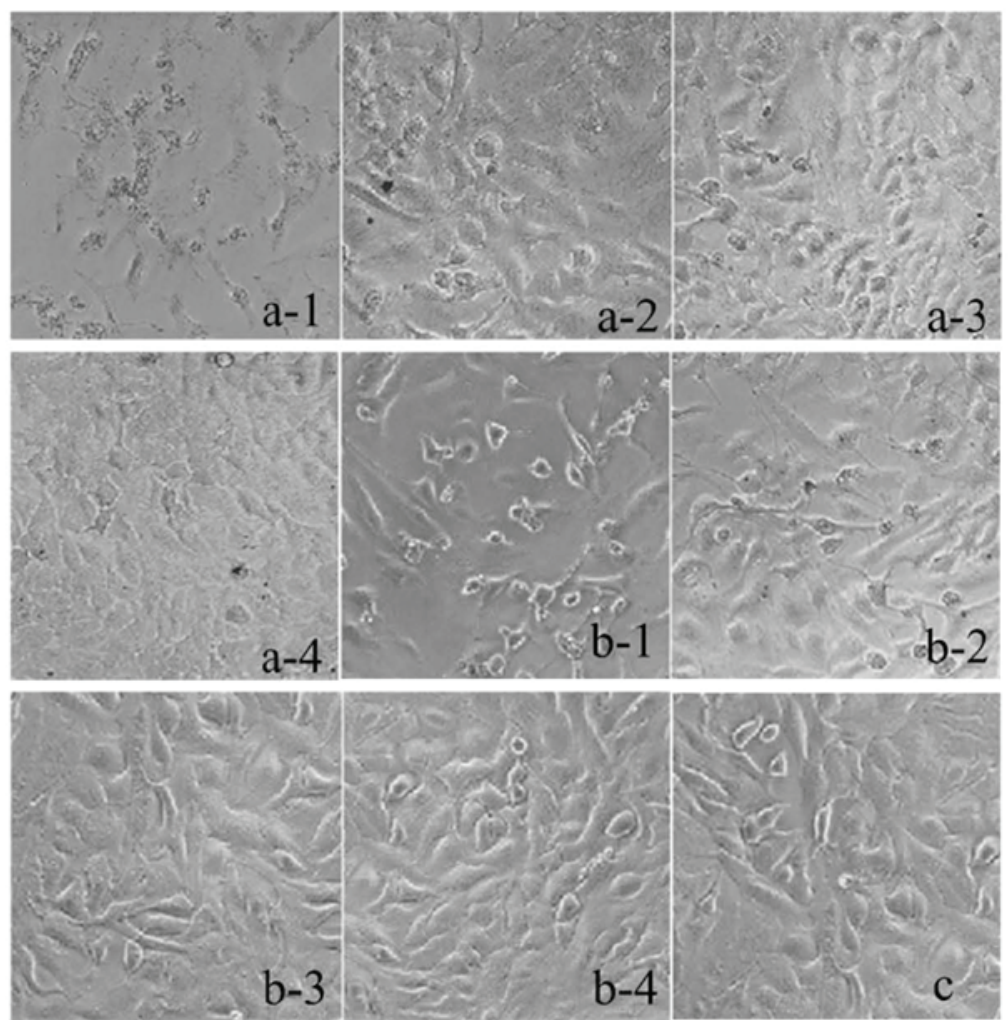

B

Growth inhibition rate of viral infected A549 cells after $\mathbf{4 8}$ h detected

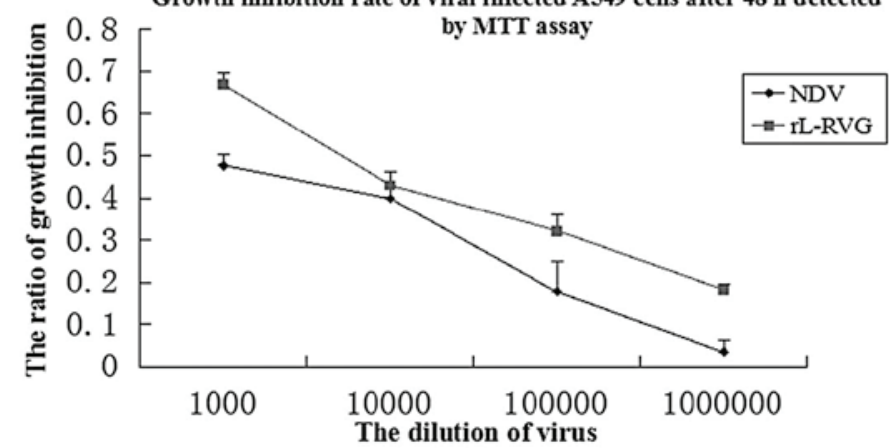

Figure 2. Morphological changes of A549 cells following infection with virus. (A) Images of A549 cells infected with virus on 48 h (magnification, $200 x$ ). a-1, $10^{-3}$ rL-RVG group; a-2, $10^{-4}$ rL-RVG group; a-3, $10^{-5}$ rL-RVG group; $44,10^{-6}$ rL-RVG group; b- $1,10^{-3}$ NDV group; b-2, $10^{-4}$ NDV group; b-3, $10^{-5}$ NDV group; b-4, $10^{-6} \mathrm{NDV}$ group; c, phosphate-buffered saline group. The adherent ability of the cells decreased. Magnification, x200. (B) Growth inhibition rate of viral infected A549 cells following 48 h determided by MTT assay. rL-RVG, LaSota NDV strain expressing rabies virus glycoprotein; NDV, Newcastle disease virus.

and the results are expressed as mean \pm standard deviation. To compare differences between multiple groups, statistical significance was analyzed using Student's t-test for data from the MTT assay, and one-way analysis of variance for TUNEL and flow cytometry data. $\mathrm{P}<0.05$ was considered to indicate a statistically significant difference between values. 
A
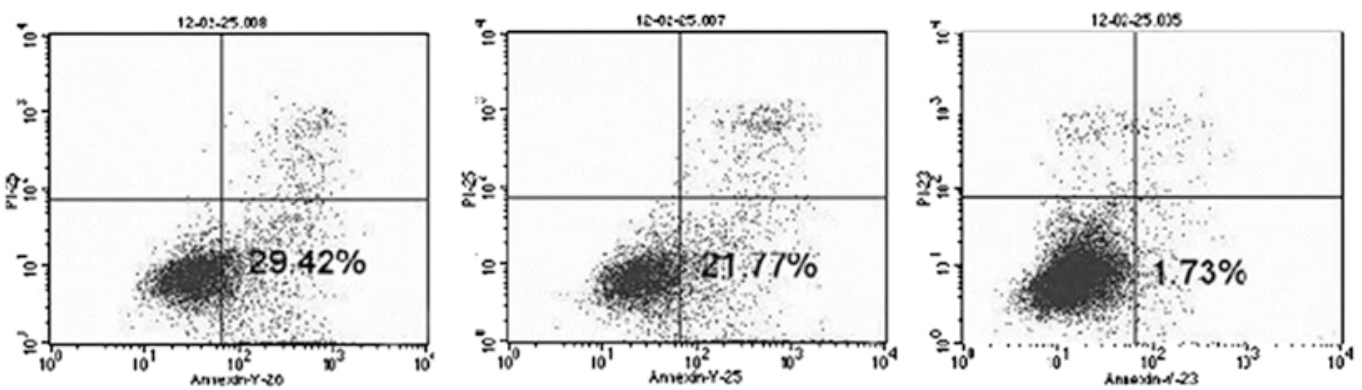

a

$\mathrm{b}$

C
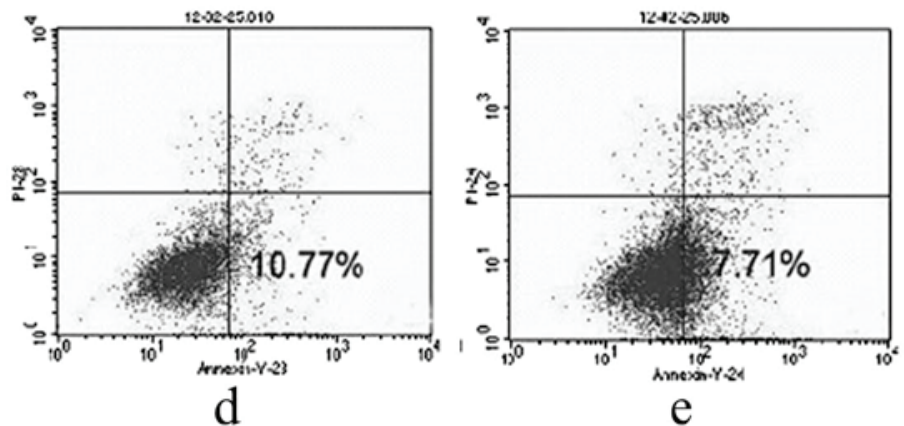

B

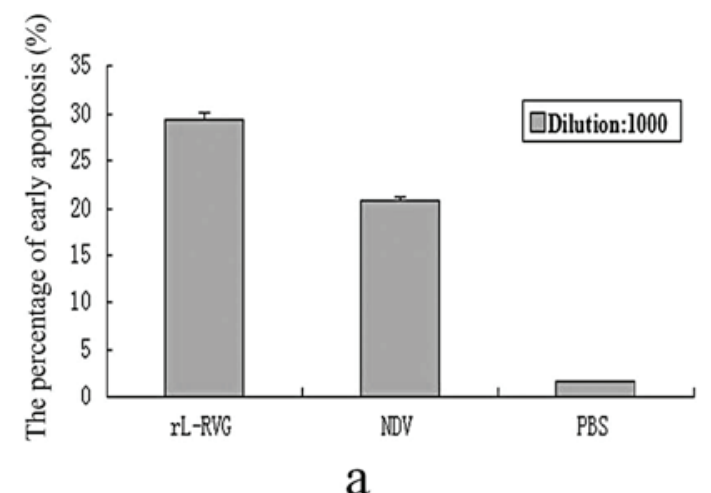

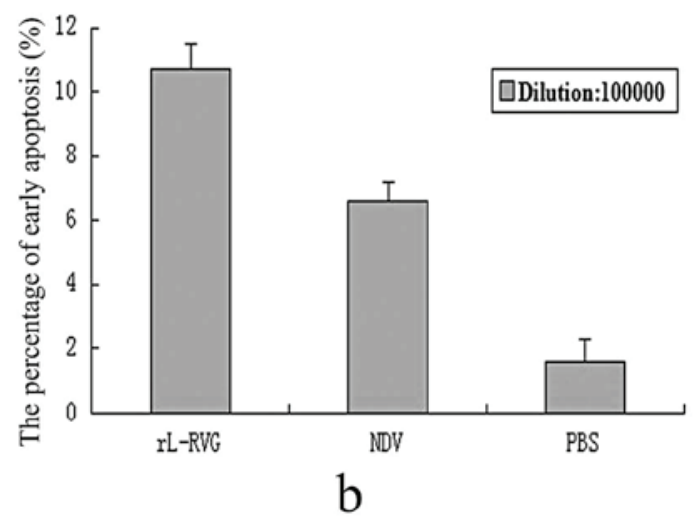

C

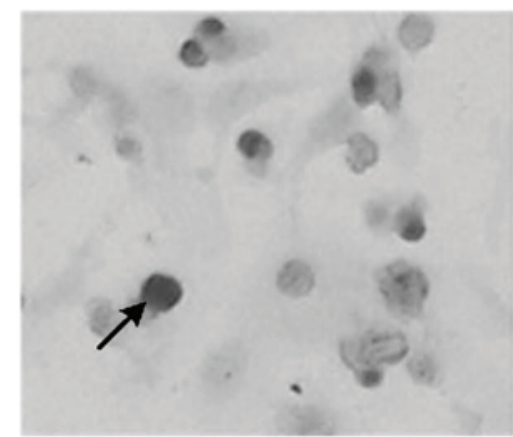

rL-RVG

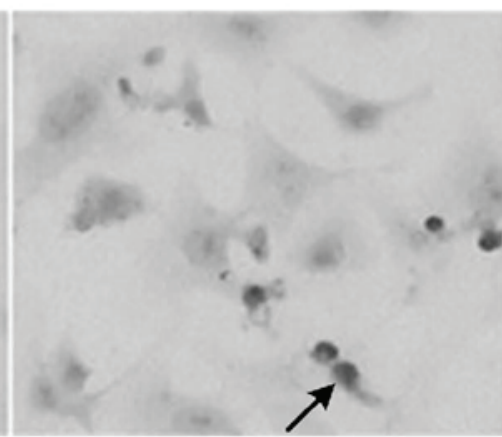

NDV

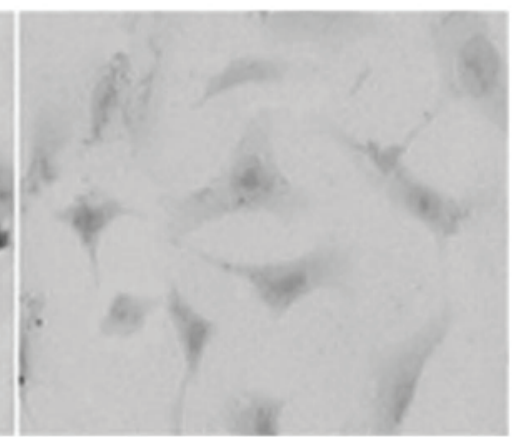

PBS

Figure 3. Apotopic effect induced by virus. (A) Flow cytometric analysis of cells in early apoptosis following $48 \mathrm{~h}$ of infection. a, rL-RVG $10^{-3}$ dilution group; $\mathrm{b}$, NDV $10^{-3}$ dilution group; c, PBS group; d, rL-RVG $10^{-5}$ dilution group; e, NDV $10^{-5}$ dilution group. (B) Quantification of early apoptotic cells from flow cytometry experiment. a, $10^{-3}$ dilution; b, $10^{-5}$ dilution. One-way analysis of variance for apoptotic cell percentages showed significant differences between rL-RVG and NDV groups $(\mathrm{P}<0.05)$. (C) Terminal deoxynucleotidyl transferase dUTP nick end labeling analysis of apoptosis in A549 cells following different infections, with arrows indicating apoptotic cells. Magnification, x200. rL-RVG, LaSota NDV strain expressing rabies virus glycoprotein; NDV, Newcastle disease virus; PBS, phosphate-buffered saline.

\section{Results}

Expression of viral genes and proteins. RT-PCR was performed to assess RVG and NDV gene expression in the A549 cells following infection with rL-RVG and NDV. Gene-specific PCR products were detected, rather than full genes. The analysis showed that the RVG gene ( 175 bp) was expressed in A549 cells following infection with rL-RVG. In the PBS and NDV groups, the RVG gene was no expressed (Fig. 1Aa). Furthermore, the NDV HN gene ( 462 bp) was expressed following infection 
Table III. Apoptotic indexes of A549 cells infected by rL-RVG and NDV.

\begin{tabular}{llll}
\hline Group & AI $(\mathrm{n}=10)$ & F-value & P-value \\
\hline rL-RVG & $0.265 \pm 0.035^{\mathrm{a}}$ & \\
NDV & $0.066 \pm 0.001^{\mathrm{b}}$ & 278.62 & \\
PBS & $0.023 \pm 0.001$ &
\end{tabular}

${ }^{a} \mathrm{rL}-\mathrm{RVG}$ versus the other two groups, $\mathrm{P}<0.01$; ${ }^{\mathrm{b} N D V}$ versus $\mathrm{PBS}$ group, $\mathrm{P}<0.05$. AI, apoptotic index; rL-RVG, LaSota NDV strain expressing rabies virus glycoprotein; NDV, Newcastle disease virus; PBS, phosphate-buffered saline.

A

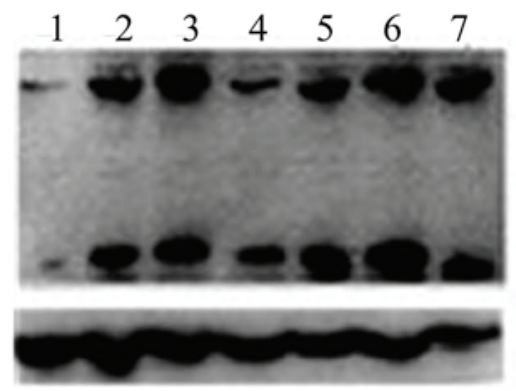

a

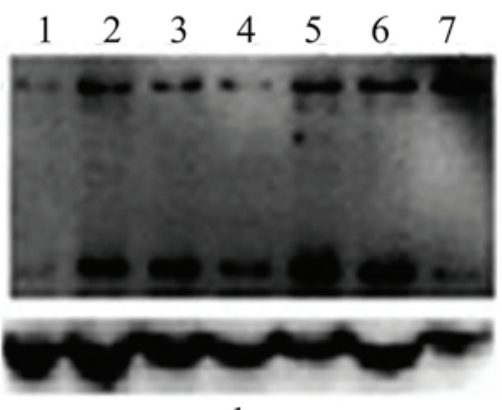

b
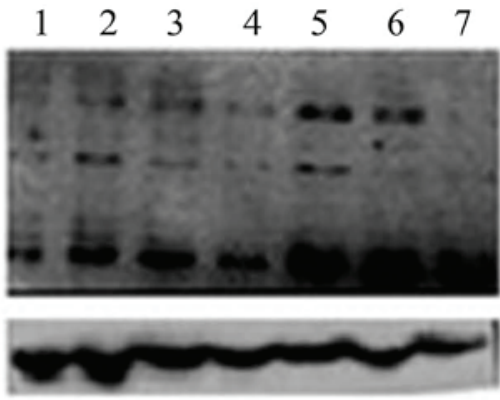

c
B

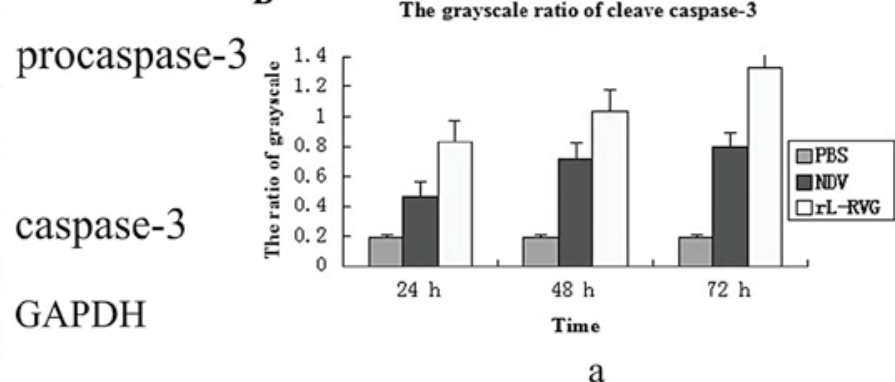

a

The grayscale ratio of cleave caspase-s

procaspase-8

caspase-8

GAPDH

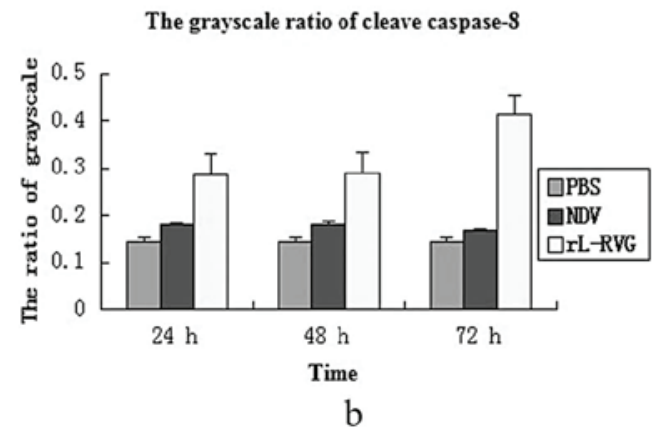

The grayscale ratio of cleave caspase- -9

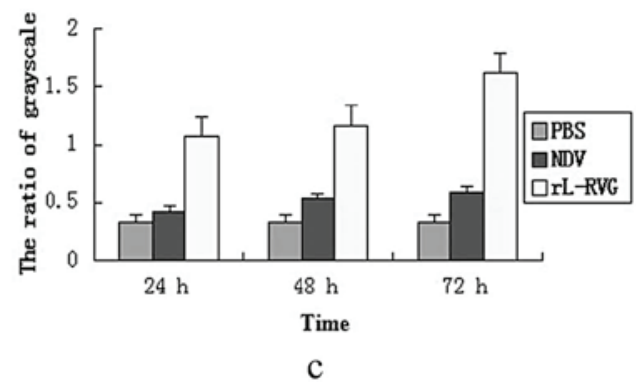

6

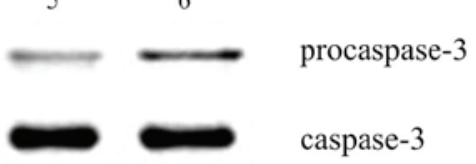

GAPDH

Figure 4. Expression of caspase induced by virus. (A) Analysis of pro-apoptotic proteins in A549 cells following infection. a, expression of caspase-3; b, expression of caspase-8; c, expression of caspase-9. 1, PBS group; 2, NDV group 24 h; 3, NDV group 48 h; 4, NDV group 72 h; 5, rL-RVG group 24 h; 6, rL-RVG group $48 \mathrm{~h} ; 7$, rL-RVG group $72 \mathrm{~h}$. (B) Grayscale ratio of caspase. a, caspase-3 expression in the rL-RVG group was significantly different from that in the other groups at any time-point. b, caspase-8 expression in the rL-RVG group was significantly different from that in the other groups, but there were no significant differences between the NDV and PBS groups. c, caspase-9 expression in the rL-RVG group was significantly different from that in the other groups at any time-point. (C) Following addition of pan-caspase inhibitor Z-VAD-FMK, the expression of the caspase-3 protein was obviously reduced. 1, PBS group; 2, NDV group; 3, rL-RVG group; 4, PBS + Z-VAD-FMK group; 5, NDV + Z-VAD-FMK group; 6, rL-RVG + Z-VAD-FMK group. Z-VAD-FMK, Z-Val-AlaAsp-fluoromethylketone; rL-RVG, LaSota NDV strain expressing rabies virus glycoprotein; NDV, Newcastle disease virus; PBS, phosphate-buffered saline. 

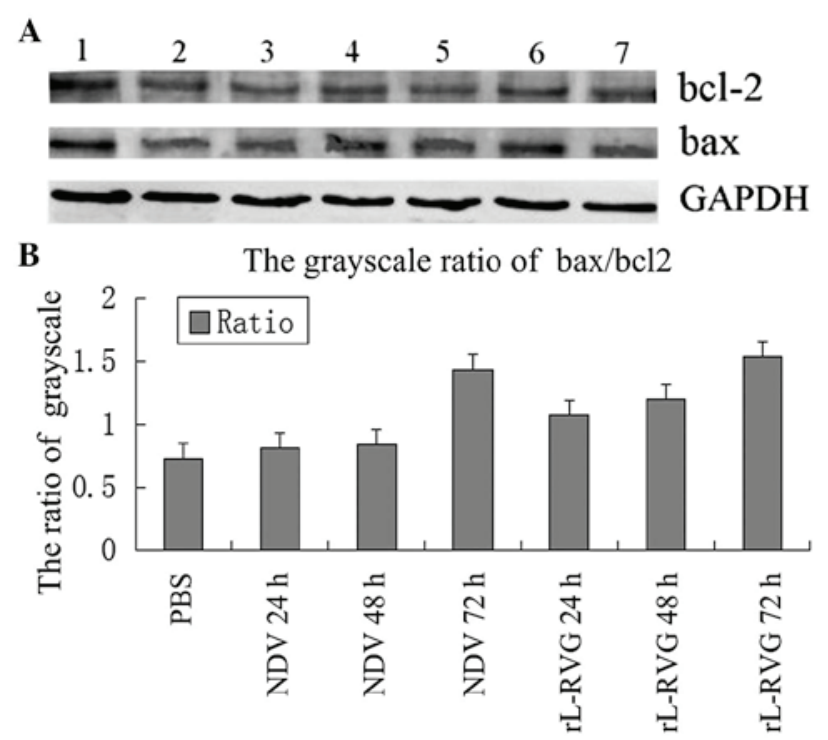

Figure 5. Expression of bax/bcl-2 in A549 cells induced by virus. (A) Expression of bax and bcl-2. 1, PBS group; 2, NDV group $24 \mathrm{~h} ; 3$, NDV group $48 \mathrm{~h} ; 4$, NDV group $72 \mathrm{~h} ; 5$, rL-RVG group $24 \mathrm{~h} ; 6$, rL-RVG group $48 \mathrm{~h} ; 7$, rL-RVG group $72 \mathrm{~h}$. (B) The bax/bcl-2 ratio of the rL-RVG group was higher than that of the NDV group at 24,48 and $72 \mathrm{~h}$ following infection. bcl-2, B-cell lymphoma 2; bax, bcl-2-associated X; rL-RVG, LaSota NDV strain expressing rabies virus glycoprotein; NDV, Newcastle disease virus; PBS, phosphate-buffered saline.

with rL-RVG and NDV. In the PBS group, the HN gene was not expressed (Fig. 1Ab). The expression levels of RVG and NDV protein were examined by western blotting and immunofluorescence assays. The results showed that RVG protein was expressed in A549 cells following infecttion with rL-RVG. However, in the PBS and NDV groups, RVG protein was no expressed. At the same time, NDV protein was detected in A549 cells following infection with rL-RVG and NDV, and none of the proteins was expressed in the PBS group (Fig. 1B and C).

Effects of the virus $r L-R V G$ on the proliferation of cancer cells. It was shown that the viability of A549 cells decreased with increasing virus concentration and incubation time following infection. rL-RVG-infected and NDV-infected cells exhibited morphological changes were observed, which were more obvious in the rL-RVG group than in the NDV group. By contrast, no changes were observed in the PBS group (Fig. 2A).

The effect of different concentrations of recombinant Newcastle disease virus rL-RVG and NDV on the growth of A549 cells following 48 and $72 \mathrm{~h}$ was assessed by MTT assay. The results showed that the inhibition rates in the rL-RVG group were significantly higher than those infected with the wild-type NDV strain, and the inhibition rate increased with time following infection. The inhibition rate of A549 cells following $48 \mathrm{~h}$ is shown in Fig. 2B and Table II.

NDVs induce apoptosis in cancer cells. Oncolytic NDV can induce apoptosis in a variety of tumor cells. In the present study, the effect of RVG expression on apoptosis induction by the recombinant virus was investigated using Annexin V/PI double staining (Fig. 3A). The percentage of cells that underwent apoptosis was higher in rL-RVG-infected than that in NDV-infected
A549 cells at $48 \mathrm{~h}$ post-infection. Of note, the percentage of cells that underwent apoptosis was significantly different between the rL-RVG and NDV groups $(\mathrm{P}<0.05)$ (Fig. 3B). The induction of A549 cell apoptosis by rL-RVG and NDV was dose- and time-dependent (data not shown). This suggested that rL-RVG was more potent in inducing tumor cell apoptosis than its parent virus, which indicated that RVG enhanced the oncolytic properties of the parental virus.

The TUNEL assay of treated A549 cells showed that the occurrence of apoptosis was easily detected following infection with rL-RVG or NDV. As shown in Table III, compared with the PBS group, the apoptotic rate was higher in the rL-RVG and NDV groups. However, compared with the NDV and PBS groups, the apoptotic rate was higher in the rL-RVG group, and there was a significant difference between the rL-RVG and NDV groups $(\mathrm{P}<0.05)$ (Fig. 3C and Table III).

NDVs increase caspase expression. The intrinsic and extrinsic pathways converge downstream at caspase-3. To examine whether the apoptosis induced by rL-RVG depends on the caspase pathway, caspase- 3 activation was assessed in A549 cells following infection with rL-RVG or NDV. As shown in Fig. 4Aa and Ba, accumulation of cleaved caspase-3 in NDV-infected A549 cells was observed at 24, 48 and $72 \mathrm{~h}$. In addition, cleaved caspase-3 levels in the rL-RVG group were higher than those in the NDV group. It was assessed whether the extrinsic apoptotic pathway was involved in tumor cells infected with rL-RVG or NDV. As illustrated in Fig. $4 \mathrm{Ab}$ and $\mathrm{Bb}$, caspase- 8 was activated at 24, 48 and $72 \mathrm{~h}$ in A549 cells infected with rL-RVG or NDV. In addition, caspase- 8 activation in the rL-RVG group was higher than that in the NDV group at 24, 48 and $72 \mathrm{~h}$. These findings demonstrated that rL-RVG infection induced the activation of caspase- 8 in tumor cells. Next, to determine the time course of induction of the initiator caspase of the intrinsic apoptotic pathway, A549 cells were infected with rL-RVG and NDV strains for 24, 48 and $72 \mathrm{~h}$, respectively. Western blot analysis was used to examine the activation of caspase-9. As shown in Fig. 4Ac and Bc, significant activation of caspase-9 was induced at 24, 48 and $72 \mathrm{~h}$. It is important that the expression of caspase-9 in the rL-RVG group was higher than that in the NDV group, which indicated that rL-RVG induced apoptosis via the intrinsic pathway and the oncolytic effects were more evident than those of NDV.

To examine whether rL-RVG-induced apoptosis depended on caspase activation, A549 cells were pretreated with the broad-specificity caspase inhibitor Z-VAD-FMK and infected by rL-RVG or NDV. The pan-caspase inhibitor Z-VAD-FMK was able to effectively inhibit rL-RVG-triggered cytolysis at $24 \mathrm{~h}$, indicating that rL-RVG-mediated cytotoxicity was caspase-dependent. This inhibitory effect was stronger than that of the NDV group, as shown in Fig. 4C.

Expression of bcl-2 and bax. To examine whether the alteration of bcl-2 and bax were induced by rL-RVG and NDV infection, the levels of bcl-2 and bax were detected by western blot analysis (Fig. 5A). The levels of bcl-2 in rL-RVG-infected and NDV-infected cells were not significantly affected at 24,48 and $72 \mathrm{~h}$. However, the expression of bax in rL-RVG-infected cells was higher than that in NDV-infected cells at 24, 48 and 
$72 \mathrm{~h}$. Bcl-2 has been shown to inhibit the bax oligomerization process. It has been proposed that the balance of the bax/bcl-2 ratio serves as a regulator of cell survival vs. death (14). The ratio of bax/bcl-2 is shown in Fig. 5B. The bax/bcl-2 ratio was significantly upregulated in rL-RVG-infected cells at 24, 48 and $72 \mathrm{~h}$. Thus, the pro-apoptotic protein dominates in the apoptotic pathway.

\section{Discussion}

Oncolytic therapy is a novel biological treatment. Oncolytic viruses can destroy harmful cells by infecting and replicating in cancer cells while leaving normal cells unaffected (15). In theory, the ability of the NDV to kill tumor cells has higher efficiency and lower side effects. Since the early 1950s, the NDV has been proven to be a potential antitumor agent with promising results in clinical trials. Numerous different studies focus on breeding effective anti-tumor NDV strains and have produced 73-T (16), PV701 (17), MTH-68/H (18), HUJ (19) and several other potential anti-neoplastic NDV strains (20). Previous studies have shown that tumor cell growth was markedly inhibited and cell apoptosis was induced by using adenovirus or fowlpox viruses as vectors to express apoptin $(21,22)$. However, these gene therapy vectors did not have inherent anti-tumor effects (23). By applying a reverse genetics technique, it is possible to produce a recombinant NDV. Compared with other vectors, NDV not only has the ability of self-replication in tumor cells, but is also is a very promising oncolytic agent. A study by Zhao et al (24) from 2008 supported the application of a recombinant NDV/IL-2 virus as an anti-tumor agent.

The recombinant Newcastle disease virus rL-RVG, which was kindly provided by the Harbin Veterinary Research Institute (Harbin, China) (13), was infected into A549 cells. PCR, western blot and immunofluorescence analysis results showed that the rL-RVG was stably expressed in A549 cells. In particular, RVG expression enabled the NDV vector to spread from cell to cell and enhance its replication in cell culture (13). Western blot analysis showed that the NDV protein bands of the rL-RVG group were stronger than those of the the NDV wild-type strain group, and immunofluorescence results showed that more NDV protein was present in the rL-RVG group than in the NDV group, which may be due to RVG protein increasing the activity of NDV. This may explain why rL-RVG had a higher oncolytic effect than the wild-type NDV. Further study is required to understand the underlying mechanism in detail.

Following infection of rL-RVG into A549 cells, cell morphology and growth were strongly affected in the in vitro experiment. The MTT assay indicated that compared with the PBS group, the rL-RVG and NDV groups exerted a toxic effect in a time- and concentration-dependent manner. Moreover, the toxic effect of rL-RVG was stronger than that of the wild-type NDV strain. It would be worthwhile exploring the underlying mechanisms leading to the different potencies of the rL-RVG and wild-type NDV in further studies.

Cell death commonly occurs due to cell necrosis and apoptosis. The TUNEL experiment demonstrated a higher apoptotic rate in the rL-RVG group as compared with that in the NDV and PBS groups. Moreover, flow cytometry results showed that compared with the PBS group, the rL-RVG and NDV groups exhibited a greater number of early apoptotic cells, and the percentage of early apoptotic cells in the rL-RVG group was higher than that in the NDV group $(\mathrm{P}<0.05)$. All the results indicated that rL-RVG induced apoptosis in A549 lung cancer cells. Therefore, it is concluded that oncolysis of of tumor cells by rL-RVG was associated with its ability to induce apoptosis.

Cell apoptosis is a basic biological function which is crucial for the body to maintain a dynamic cell balance and stability in the tissue. Studies have revealed delineation of apoptotic pathways activated in NDV-infected cells (25). Multiple studies have investigated the mechanisms underlying NDV-induced apoptosis. NDV can induce apoptosis by activating both extrinsic and intrinsic apoptotic pathways (26). Elankumaran et al (27) have shown that in tumor cells infected with NDV, apoptosis was triggered via the extrinsic pathway, which was induced by caspase- 8 activation (27). However, such processes do not occur in all tumor cells. In the present study, cleavage of caspase- 8 was also detected in the rL-RVG and NDV groups. Moreover, levels of caspase- 8 in the rL-RVG group were higher than those in the wild-type NDV group. It may therefore be suggested that compared with the NDV strain, rL-RVG induced stronger extrinsic apoptosis. At the same time, intrinsic apoptotic pathways were activated in the rL-RVG and NDV groups, which were induced by caspase-9 activation. The caspase-3 inhibitor Z-VAD-FMK was able to effectively inhibit NDV-triggered cytolysis following $24 \mathrm{~h}$. This demonstrated that rL-RVG-mediated and NDV-mediated cytotoxicity were caspase-dependent, and that rL-RVG induced higher levels of apoptosis than NDV.

By changing the outer membrane permeability and mitochondrial activation, members of the bcl-2 protein family were regulated to control cell apoptosis. The bcl-2 family members can be divided into two categories according to their structure and function: (1) Apoptosis-promoting members, such as Bax, which have the ability to promote the opening of the mitochondrial permeability transition pore, releasing caspase and promoting cell apoptosis (28); and (2) inhibitors of apoptosis, such as bcl-2, which protect the integrity of the mitochondrial outer membrane and thereby prevent cytochrome $\mathrm{C}$ release. Western blot analysis showed that the expression of anti-apoptotic bcl-2 in the rL-RVG group did not change, but bax expression was increased. Bcl-2 inhibits the bax oligomerization process, and the ratio of bax/bcl-2 regulates cell survival vs. death (14). The bax/bcl-2 ratio was higher in the rL-RVG group than that in the NDV group at 24, 48 and 72 h. Accordingly, whether the involvement of viral protein or soluble intermediate factors was responsible for the upregulation of bax and downregulation of bcl-2 expression requires further investigation.

The anti-cancer activity of rL-RVG in lung cancer may have additional underlying mechanisms. For instance, the 198-214 amino acid sequence of rabies virus glycoprotein is highly homologous with the $30-56$ sequence in $\lambda$-bungatotoxin, which is able to combine with nicotinic acetylcholine receptors (nAChRs). nAChRs are considered to be combined with the rabies virus glycoprotein. Plummer et al (29) showed that $\alpha 7-n$ AChR was widely expressed in lung cancer cells, including squamous carcinoma, adenocarcinoma, large cell 
carcinoma and small cell carcinoma. This therefore suggested that rL-RVG may be able to be absorbed by tumor cells due to its affinity to $\alpha 7$-nAChR provided by RVG.

In conclusion, rL-RVG is a novel type of oncolytic virus and has a higher oncolytic efficacy than the previously reported wild-type NDV. The underlying mechanism of action of the recombinant $\mathrm{rL}-\mathrm{RVG}$ strain proceeded via the induction of apoptosis in NSCLC A549 cells. This induction of apoptosis was a caspase-dependent process. The present study provided a strong foundation for vaccine studies; however, the detailed mechanism underlying the anti-cancer activity of rL-RVG requires further study. The present study provided strong evidence for the potential of rL-RVG to be used for clinical treatment of cancer.

\section{Acknowledgements}

The authors would like to thank Professor Zhigao $\mathrm{Bu}$ and Dr Jinying Ge from the State Key Laboratory of Veterinary Biotechnology, Harbin Veterinary Research Institute, Chinese Academy of Agricultural Sciences, Harbin, China for suggestions regarding the experiments performed. The authors would also like to thank Dr Yan Tao and Aihua Gong from Jiangsu University (Jiangsu, China) for kindly providing technical support.

\section{References}

1. Jemal A, Tiwari RC, Murray T, et al; American Cancer Society: Cancer statistics, 2004. CA Cancer J Clin 54: 8-29, 2004.

2. DeSantis C, Naishadham D and Jemal A: Cancer statistics for African Americans, 2013. CA Cancer J Clin 63: 151-166, 2013.

3. Zhang J: The treatment of lung carcinoma in aged people. Chin J Geriatr 4: 316-318, 2001.

4. Vähä-Koskela MJ, Heikkilä JE and Hinkkanen AE: Oncolytic viruses in cancer therapy. Cancer Letters 254: 178-216, 2007.

5. Lam HY, Yeap SK, Rasoli M, et al: Safety and clinical usage of newcastle disease virus in cancer therapy. J Biomed Biotechnol 2011: 718710, 2011.

6. Yusoff K and Tan WS: Newcastle disease virus: macromolecules and opportunities. Avian Pathol 30: 439-455, 2001.

7. Römer-Oberdörfer A, Werner O, Veits J, Mebatsion T and Mettenleiter TC: Contribution of the length of the HN protein and the sequence of the $\mathrm{F}$ protein cleavage site to Newcastle disease virus pathogenicity. J Gen Virol 84(Pt 11): 3121-3129, 2003.

8. Wu Y, Zhang X, Wang X, Wang L, Hu S, Liu X, Meng S: Apoptin enhances the oncolytic properties of Newcastle disease virus. Intervirology 55: 276-86, 2012.

9. Silverstein SC and Marcus PI: Early stages of newcastle disease virus-hela cell interaction: an electron microscopic study. Virology 23: 370-380, 1964.

10. Johnson N, McElhinney LM, Smith J, Lowings P and Fooks AR: Phylogenetic comparison of the genus lyssavirus using distal coding sequences of the glycoprotein and nucleoprotein genes. Arch Virol 147: 2111-2123, 2002.
11. Préhaud C, Lay S, Dietzschold B and Lafon M: Glycoprotein of nonpathogenic rabies viruses is a key determinant of human cell apoptosis. J Virol 77: 10537-10547, 2003.

12. Ge J, Wang X, Tao L, et al: Newcastle disease virus-vectored rabies vaccine is safe, highly immunogenic, and provides long-lasting protection in dogs and cats. J Virol 85: 8241-8252, 2011.

13. Reed L J and Muench H: A simple method of estimating fifty percent endpoints. Am J Hyg 27: 493-497, 1938.

14. Molouki A, Hsu YT, Jahanshiri F, Rosli R and Yusoff K: Newcastle disease virus infection promotes bax redistribution to mitochondria and cell death in HeLa cells. Intervirology 53: 87-94, 2010.

15. Ali R, Alabsi AM, Ali AM, et al: Cytolytic effects and apoptosis induction of Newcastle disease virus strain AF2240 on anaplastic astrocytoma brain tumor cell line. Neurochem Res 36: 2051-2062, 2011.

16. Batliwalla FM, Bateman BA, Serrano D, et al: A 15-year follow-up of AJCC stage III malignant melanoma patients treated postsurgically with Newcastle diseasevirus (NDV) oncolysate and determination of alterations in the CD8 T cell repertoire. Mol Med 4: 783-794, 1998.

17. Pecora AL, Rizvi N, Cohen GI, et al: Phase I trial of intravenous administration of PV701, an oncolytic virus, in patients with advanced solid cancers. J Clin Oncol 20: 2251-2266, 2002.

18. Csatary LK, Moss RW, Beuth J, Töröcsik B, Szeberenyi J, Bakacs T: Beneficial treatment of patients with advanced cancer using a Newcastle disease virus vaccine (MTH-68/H). Anticancer Res 19: 635-638, 1999.

19. Freeman AI, Zakay-Rones Z, Gomori JM, et al: Phase I/II trial of intravenous NDV-HUJ oncolytic virus in recurrent glioblastoma multiforme. Mol Ther 13: 221-228, 2006

20. Ravindra PV, Tiwari AK, Sharma B and Chauhan RS: Newcastle disease virus as an oncolytic agent. Indian J Med Res 130: 507-513, 2009.

21. Li X, Jin N, Mi Z, et al. Antitumor effects of a recombinant fowlpox virus expressing apoptin in vivo and in vitro. Int $\mathrm{J}$ Cancer 119: 2948-2957, 2006.

22. Pietersen AM, van der Eb MM, Rademaker HJ, et al: Specific tumor-cell killing with adenovirus vectors containing the apoptin gene. Gene Ther 6: 882-892, 1999.

23. Wu Y, Zhang X, Wang X, et al: Apoptin enhances the oncolytic properties of Newcastle disease virus. Intervirology 55: 276-286, 2012.

24. Zhao H, Janke M, Fournier P and Schirrmacher V: Recombinant Newcastle disease virus expressing human interleukin-2 serves as a potential candidate for tumor therapy. Virus Res 136: 75-80, 2008.

25. Ravindra PV, Tiwari AK, Ratta B, et al: Induction of apoptosis in Vero cells by Newcastle disease virus requires viral replication, de-novo protein synthesis and caspase activation. Virus Res 133: 285-90, 2008.

26. Zamarin D and Palese P: Oncolytic Newcastle disease virus for cancer therapy: old challenges and new directions. Future Microbiol 7: 347-367, 2012.

27. Elankumaran S, Rockemann D and Samal SK: Newcastle disease virus exerts oncolysis by both intrinsic and extrinsic caspase-dependent pathways of cell death. J Virol 80: 7522-7534, 2006.

28. Hirsch T, Marzo I and Kroemer G: Role of the mitochondrial permeability transition pore in apoptosis. Biosci Rep 17: 67-76, 1997.

29. Plummer HK III, Dhar M and Schuller HM: Expression of the alpha7 nicotinic acetylcholine receptor in human lung cells. Respir Res 6: 29, 2005. 